\title{
Neonates and medicines: a roadmap to further improve neonatal pharmaceutical care
}

\author{
Karel Allegaert ${ }^{1,2,3} \cdot$ Catherine Sherwin ${ }^{4,5}$
}

Received: 14 December 2015 / Accepted: 28 December 2015 /Published online: 7 January 2016

(C) Springer-Verlag Berlin Heidelberg 2016

The treatment of newborns with safe and effective medicines is of critical importance for their outcome and subsequent quality of life. Despite this, it is still a common practice to prescribe medicines to neonates outside the label, extrapolating from dosing regimens and indications validated in older populations and based on non-neonatal pathophysiology. In a recent meta-analysis (2015) evaluating 829 (1994-2012) studies on prescribing practices in pediatric hospital care, off-label and unlicensed medicines prescriptions ranged from 12 to $71 \%$ and 0.2 to $48 \%$. These authors hereby reconfirmed that (pre)term neonates were still most commonly exposed to off-label and unlicensed medicines [12].

The federal US legislation and similar European initiatives have resulted in a relevant increase in pharmacological studies in children, with a subsequent significant increase in label changes. Unfortunately, too few included drug label changes

Communicated by Patrick Van Reempts

Both authors contributed to the text and agreed on the final version.

Karel Allegaert

allegaertkarel@hotmail.com

1 Intensive Care and Department of Surgery, Erasmus MC-Sophia Children's Hospital, Rotterdam, The Netherlands

2 Department of Development and Regeneration, KU Leuven, Belgium

3 Neonatal Intensive Care Unit, University Hospitals Leuven, Herestraat 49, 3000 Leuven, Belgium

4 Division of Clinical Pharmacology, Department of Pediatrics, University of Utah, Salt Lake City, UT, USA

5 Clinical Trials Office, Department of Pediatrics, University of Utah, Salt Lake City, UT, USA specific to neonates. To further illustrate this, there were 406 pediatric label changes (Food and Drug Administration, 1997-2010), but only 23 drugs resulted in 11 labeled indications (e.g., linezolid, rocuronium, remifentanil, sevoflurane, stavudine, nevirapine) for neonates. The absence of label change was most commonly because of unproven efficacy, despite the fact that these compounds (e.g., paracetamol, caspofungin, valganciclovir) are likely relevant for neonates [14]. This may largely reflect the difficulties to proof efficacy due to heterogeneity in patients, variability in outcome variables, and uncertainty on biomarkers, in addition to demonstrating safety (risk of adverse drug events) in neonates. Despite the significant improvements in the knowledge on pharmacotherapy in older children, neonates still remain therapeutic orphans $[2,14]$.

In this issue of the journal, Campino et al. report on the rate of errors (calculation or accuracy) in intravenous medicine preparations when performed either bedside in 10 Spanish neonatal intensive care units (NICUs) or in hospital pharmacy (HP) services. The study group hereby evaluated the impact of a structured intervention (protocol standardization, education) on the number of errors and documented significant improvements in both calculation errors (1.35 to $0 \%)$ and accuracy (54.7 and $38.3 \%$ to 38.3 and $14.6 \%$ in NICU and HP, respectively) [6]. This intervention paper follows on a previous paper which described a pre-intervention phase [5] and illustrates the potential impact of preventive strategies on the extent of drug errors.

Development of a roadmap to improve neonatal pharmaceutical care should aim to improve the knowledge on clinical pharmacology, clinical pharmacy, and approaches to improve the knowledge and use of neonatal pharmacovigilance initiatives [1]. Another keystone component of such a roadmap should focus on neonatal drug development, i.e., drug development driven by neonatal pathophysiology (Fig. 1). 
Fig. 1 A roadmap to improve neonatal pharmaceutical care include improvements in the fields of clinical pharmacology $[1-4,16]$, clinical pharmacy $[5,6$, $10,11,13,15]$, and pharmacovigilance $[7,8]$ in neonates, but should also shift towards medicines and product development driven by neonatal pathophysiology [9]

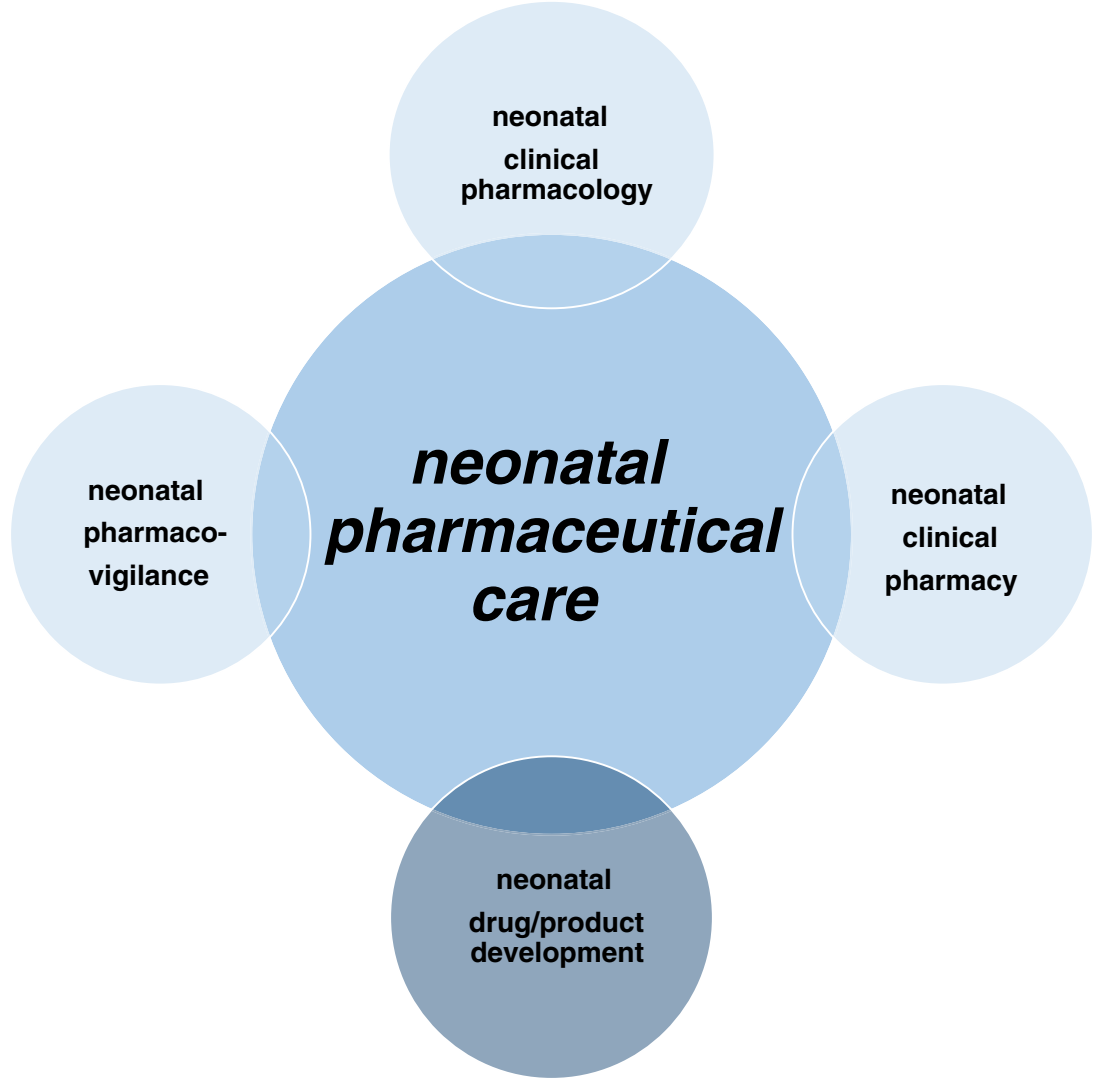

\section{Neonatal clinical pharmacology: limited in size, extensive in variability}

The aim of administering a given compound is to reach effective treatment for a specific disease while avoiding disproportional side-effects and adverse events. Clinical pharmacology aims to predict drug-specific (side)-effects based on pharmacokinetics (PK) and pharmacodynamics (PD). PK (e.g., absorption, distribution, and elimination, through either metabolism or primary renal elimination) hereby estimates the relationship between a drug concentration at a specific site (e.g., plasma or cerebrospinal fluid) and time ("what the body does to the drug"). PD describes the concentration effect relationship ("what the drug does to the body") [1]. Requiring specific consideration is that these maturational changes in physiology are most prominent in early infancy and are highly variable between preterm and term neonates. These maturational physiological trends can be additionally further affected by either treatment modalities (e.g., whole body cooling, extracorporeal membrane oxygenation (ECMO), or pharmacotherapy), pathophysiological processes or co-morbidities (e.g., perinatal asphyxia, sepsis, renal failure, patent ductus arteriosus). All these changes, both maturational (e.g., age, weight) and pathophysiological can result in extensive variability in dosing within the first days and months of postnatal life [1]. Knowledge integration through utilization of pharmacokinetic modeling is a method that could improve the current situation. Such predictive models may convert neonatal pharmacotherapy from explorative to confirmatory $[3,4,16]$.

\section{Neonatal clinical pharmacy: how to cope with neonatal formulation requirements}

The relevance of formulation issues throughout pediatrics, including neonates has been recently been highlighted, also in this journal $[11,15]$. The administration of drugs via all routes to neonates poses significant challenges. This is even more evident for intravenous medicines. These challenges arise from slow intravenous flow rates, small drug volume, dead space volume, excipients, and limitations on the flush volume that can be given in neonates. An appreciation of the substantial delay and variability in the rate of drug delivery from the intravenous line is often lacking, but the available knowledge on this aspect has recently been summarized [13]. Knowledge on neonatal drug delivery has advanced, but there is still a deficiency of technologies and formulations developed specifically for this population. As a recent illustration, we refer to the evaluation of the use of uncoated mini-tablets instead of syrup for neonates. Uncoated mini-tablets offer the potential of a single formulation for different age groups and 
avoid the need for specific excipients or taste-masking compounds $[10,15]$.

\section{Neonatal pharmacovigilance: how to recognize the signal in the noise?}

Neonatal pharmacovigilance is a powerful tool to improve healthcare outcomes, but there is an obvious and urgent need to improve our practices through tailored adverse drug reaction (ADR) reporting, prevention, and management. As it is an accepted and common practice to prescribe unlicensed and off-label drugs in neonates, a reasonable and relevant description for an ADR may be "an unintended, but harmful effect resulting from the administration to medication(s) intended for diagnostic or therapeutic reasons." Such a definition also covers medication errors (e.g., wrong dose, wrong route, or wrong patient) [7]. In addition to the lack of labeling (e.g., eminence based practices), inappropriate formulations (e.g., highly concentrated, excipients), (poly)pharmacy (e.g., maturational drug-drug interactions), immature organ function (how to discriminate between normal maturational function and toxicity) and multiple illnesses further increase the risks for ADRs in neonates.

Pharmacovigilance needs to be adapted to take into consideration the characteristics that are specific to neonates. Such a strategy should be based on prevention through drug prescription and administration errors prevention (e.g., formulation, bedside manipulation, access) strategies, detection through laboratory, or clinical outlier data signaling (e.g., reference laboratory values, overall high morbidity), and subsequent assessment. Both papers of Campino et al. fit perfectly in such a preventive strategy $[5,6]$. However, once a signal has been detected, the differentiation of "true" ADRs from confounding events associated with immaturity, organ dysfunction, or underlying diseases remains difficult. An algorithm as suggested by Du et al. based on 13 questions tailored to neonates has been shown to be more reliable when compared to the Naranjo approach [8].

\section{Up to the next level: product development driven by neonatal pathophysiology}

In addition to the above discussed issues, there is also a more fundamental aspect. At present, almost all compounds administered or evaluated in neonates were initially developed for other populations, with subsequent tailoring to the characteristics of neonates (e.g., formulation, similar or different indication). A product development plan driven by a newbornfocused research agenda with specific consideration on their pathophysiology (e.g., surfactant for hyaline membrane disease) has the potential to lead to major breakthroughs and advancements in neonatal pharmaceutical care. Recently, the International Neonatal Consortium (INC) has been developed as part of the Critical Path Initiative to serve as a forum for the neonatal community to develop consensus statements (e.g., standardization of methods, standard of care consensus statements, population specific biomarkers, modeling approaches, trial designs, clinical outcome assessment tools, formulation issues) with the ultimate goal to improve neonatal medicines development [9]. This initiative explicitly aims to accelerate the availability of safe and effective therapies for neonates. As a first step, a research-focused research agenda has recently been put forward, to cover prevention and treatment of neonatal brain injury (e.g., neonatal seizures), lung injury (e.g., bronchopulmonary dysplasia), gastro-intestinal injury (e.g., necrotizing enterocolitis), neonatal sepsis, retinopathy of prematurity, and neonatal abstinence syndrome [9]. Although this may look ambitious and strategic, far away from bedside care, other examples within the Critical Path Initiative [9] have proven to be very effective to improve pharmacotherapy for specific disease entities.

In conclusion, improvement in the pharmaceutical care in neonates is much needed, but possible using the right frameworks. Knowledge integration and translation to clinical practice is crucial and must consider clinical pharmacology, clinical pharmacy, or pharmacovigilance (Fig. 1). Similar to both papers of Campino et al., healthcare providers involved in neonatal care should be encouraged to consult and assess the available evidence and subsequently explore effective interventions. In addition, all stakeholders involved (healthcare providers, academia, agencies, and sponsors) should consider to product development driven by a newborn-focused agenda with emphasis on their pathophysiology. Improved knowledge could and should result in major progresses in neonatal pharmaceutical care.

Acknowledgments The clinical research of K Allegaert is supported by the Fund for Scientific Research, Flanders (fundamental clinical investigatorship $1800214 \mathrm{~N}$ ) and further facilitated by the agency for innovation by Science and Technology in Flanders (IWT) through the SAFEPEDRUG project (IWT/SBO 130033)

\section{References}

1. Allegaert K, Langhendries JP, van den Anker JN (2013) Educational paper: do we need neonatal clinical pharmacologists ? Eur J Pediatr 172:429-435

2. Allegaert K, van den Anker J (2015) Neonatal drug therapy: the first frontier of therapeutics for children. Clin Pharmacol Ther 98: 288-297

3. Anderson BJ, Allegaert K, Holford NH (2006) Population clinical pharmacology of children: general principles. Eur J Pediatr 165: 741-746

4. Anderson BJ, Allegaert K, Holford NH (2006) Population clinical pharmacology of children: modelling covariate effects. Eur J Pediatr 165:819-829 
5. Campino A, Arranz C, Unceta M, Rueda M, Sordo B, Pilar P et al (2015) Medicine preparation errors in ten Spanish neonatal intensive care units. Eur J Pediatr. doi:10.1007/s00431-015-2615-4

6. Campino A, Santesteban E, Pascual P, Sordo B, Arranz C, Unceta $M$, et al (2016) Strategies implementation to reduce medicine preparation error rate in neonatal intensive care units. Eur J Pediatr (in press, accepted)

7. Choonara I (2013) Educational paper: aspects of clinical pharmacology in children - pharmacovigilance and safety. Eur J Pediatr 172:577-580

8. Du W, Lehr VT, Lieh-Lai M, Koo W, Ward RM, Rieder MJ et al (2013) An algorithm to detect adverse drug reactions in the neonatal intensive care unit. J Clin Pharmacol 53:87-95

9. International Neonatal Consortium. Accelerating the development of safe and effective therapies for neonates. Critical Path Institute. http://c-path.org/programs/inc. Accessed 06 December 2015

10. Klingmann V, Seitz A, Meissner T, Breitkreutz J, Moeltner A, Bosse HM (2015) Acceptability of uncoated mini-tablets in neonates - a randomized controlled trial. J Pediatr 167:893-896.e2
11. Linakis MW, Roberts JK, Lala AC, Spigarelli MG, Medlicott NJ, Reith DM et al (2015) Challenges associated with route of administration in neonatal drug delivery. Clin Pharmacokinet. doi:10. 1007/s40262-015-0313-Z

12. Magalhaes J, Rodrigues AT, Rogue F, Figueiras A, Falcao A, Herdeiro MT (2015) Use of off-label and unlicensed drugs in hospitalized paediatric patients: a systematic review. Eur J Clin Pharmacol 71:1-13

13. Sherwin CM, Medlicott NJ, Reith DM, Broadbent RS (2014) Intravenous drug delivery in neonates: lessons learnt. Arch Dis Child 99:590-594

14. Stiers JL, Ward R (2014) Newborns, one of the last therapeutic orphans to be adopted. JAMA Pediatr 168:106-108

15. Tuleu C, Breitkreutz J (2013) Educational paper: formulationrelated issues in pediatric clinical pharmacology. Eur $\mathrm{J}$ Pediatr 172:717-720

16. Vinks AA, Emoto C, Fukuda T (2015) Modeling and simulation in pediatric drug therapy: application of pharmacometrcis to define the right dose for children. Clin Pharmacol Ther 98:298-308 